\title{
Application of partial differential equations in image processing
}

\author{
Xiaoke Cui ${ }^{1, a^{*}}$ \\ ${ }^{1}$ Pingdingshan Industrial College of Technology, Pingdingshan, Henan, 467001, China \\ aemai:cuixiaoke111@126.com
}

Keywords: Structure tensor; Nonlinear edge enhancement; Image denoising; Nonlocal operator.

\begin{abstract}
Based on the superiority of PDE and its physical mechanism analysis from image processing, combining variational methods, functional analysis, differential geometry, projective geometry and other mathematical tools to the image denoising smoothing, image segmentation, image restoration, image to enlarge, image reconstruction, medical image processing, remote sensing image processing and other fields are widely used. Especially nonlinear diffusion equation has a very important role in image processing and machine vision. This paper focuses on the theme of image de-noising and enhancement of several PDE-based image denoising and enhancement model for analysis and improvement. It discusses the anisotropic diffusion method causes agglomeration and some solutions, and diffusion equation with a nonlinear structure tensor combined spread function and select the appropriate parameters to establish an improved model for image edge enhancement. While the model spread function and the key parameters were discussed, and numerical experiments to verify the feasibility of the method.
\end{abstract}

\section{Introduction}

In recent years, based on the image processing method called partial differential equations and the development of image processing is an important branch, and associated image processing content with the benefits of co-researchers in related fields to become the focus of attention [1-3]. The earliest work dates back to Nagao, Rudin and so on smooth image and image enhancement Koenderink research and exploration of image structure [4]. In addition, image processing and other branches of mathematics, such as image level set image shape and morphology, also injected some life into the contents and the formation of disciplines. Especially image processing directly affect two important branches of the discipline eventually formed: one image segmentation, two image filtering $[5,6]$.

Currently PDE image denoising and enhancement using mainly a theoretical model based on three aspects: First, the model is axiomatic theory of partial differential equations based on multi-scale analysis [7]. Such methods include non-linear direction from the direction of the angle of the filter analysis and design diffusion equation [8]. This method can be automatically adjusted according to the local diffusion coefficient of the image feature information, in order to achieve diffusion of adaptive filtering. But this partial differential equation model modular redundancy exists, and existence and uniqueness is one step to get into the discussion of research $[9,10]$.

This paper focuses on the theme of image de-noising and enhancement of several PDE-based image denoising and enhancement model for analysis and improvement. It discusses the anisotropic diffusion method causes agglomeration and some solutions, and diffusion equation with a nonlinear structure tensor combined spread function and select the appropriate parameters to establish an improved model for image edge enhancement. While the model spread function and the key parameters were discussed, and numerical experiments to verify the feasibility of the method.

\section{Static grayscale images of discrete}

Mathematically speaking, an image available to an energy distribution function E, where $\mathrm{y}, \mathrm{z}$ coordinate space represents pixel, $y$ is the light wavelength, $f$ denotes asked. In particular, the brightness of a still image on a plane can be expressed as [11]: 


$$
f(x, y)=\in \int_{0}^{+\infty} E(x, y, \lambda) S(\lambda) d \lambda
$$

Wherein, $\mathrm{E}(\mathrm{x}, \mathrm{y}, \mathrm{z})$ is the radiation energy of the objective things, $\mathrm{S}(\mathrm{La})$ for units A wavelength of radiant energy produced equivalent brightness, i.e. luminance sensitivity function [12].

Under normal circumstances, the image on the spatial and luminance values are continuous Geng, known as a continuous image or simulation image, if successive discrete image, it would be a digital image on the spatial and brightness, which it is a You can form an image by computer processing. Digital images can be represented as a matrix:

$$
\begin{gathered}
F=\left(f_{x, y}\right)_{M \times N} \\
F=[f(1,1), f(1,2), \mathrm{L}, f(1, N), \mathrm{L}, f(M, 1), f(M, 2), \mathrm{L}, f(M, N)]
\end{gathered}
$$

Image processing technology content is very rich, depending on the degree of abstraction and research methods may be divided into three levels: image processing, image analysis and image understanding. Low-level image processing emphasizes the transition between images. All the contents described herein are all low-level image processing. In general, low-level image processing is originally acquired input image, the output is to improve the quality or some more suitable for certain applications of the image, which is output to the image analysis is often the middle. Low-level image processing and two very important elements: image enhancement and image restoration. The general theory is that it does not exist. Its purpose is to deal with the visual interpretation, the final judgment by the observer effect of a particular method, which is a kind of image processing means for processing a strong subjective.

Image enhancement, image restoration and image segmentation is the image processing and analysis of the main problems, image smoothing and edge detection is a major direction. In the image the image noise using a low-pass filtering noise will appear blurred boundaries, while the edges are precisely preserved a lot of information. The classic image denoising methods, such as Gaussian filtering, median filtering and other major high-frequency component of the image is removed, while the details of the image also includes an edge in the high frequency part of the branches, so often there will also be blurred edge image off phenomenon. Impact of noise on the image is multifaceted, so noise image model also has many types.

\section{Denoising partial differential model}

Objective world objects in their time due to the different observations of the selected dimensions, thus different forms. The human eye in the picture is always observed from coarse to fine, which is the first on the scene to get a rough outline about the scale, and then gradually refined.

Based image processing from the point of view of partial differential equations of motion Cambodia image processing, the initial value problem originated in the heat conduction equation:

$$
\left\{\begin{array}{l}
\frac{\partial u}{\partial t}=\Delta u \\
u(x, y, 0)=u_{0}(x, y)
\end{array}(x, y) \in R^{2}\right.
$$

Solutions of this equation can be expressed as a Gaussian function with ".G, y) convolution, namely:

$$
\begin{gathered}
u(x, y, t)=G_{\sigma}(x, y) * u_{0}(x, y) \\
G_{\sigma}(x, y)=\frac{1}{\sqrt{2 \pi} \sigma} \exp \left[-\left(x^{2}+y^{2}\right) / 4 \sigma\right]
\end{gathered}
$$


It is a Gaussian function, where a representative of a scale parameter. $\triangle$ "is on spatial variables the Laplace operator. The noise removal process using the diffusion equation, which cover witkin to a continuous change from $\mathrm{O}$ to $\infty$, we proposed the concept of scale space.

Energy functional is:

$$
E(u)=\frac{1}{2} \int|\nabla u|^{2} d \Omega
$$

This equation can not be maintained when the denoising characteristic boundary image. The reason is the Laplace operator of linearity, the deeper reason is the image of energy functional $\mathrm{E}$ missing items holding structure.

\section{Results and discussion}

The flow chart of texture image recognition based on Differential Evolution Probabilistic Neural Network (DEPNN) algorithm is got from the algorithm in document 10 and shown in Figure 1.

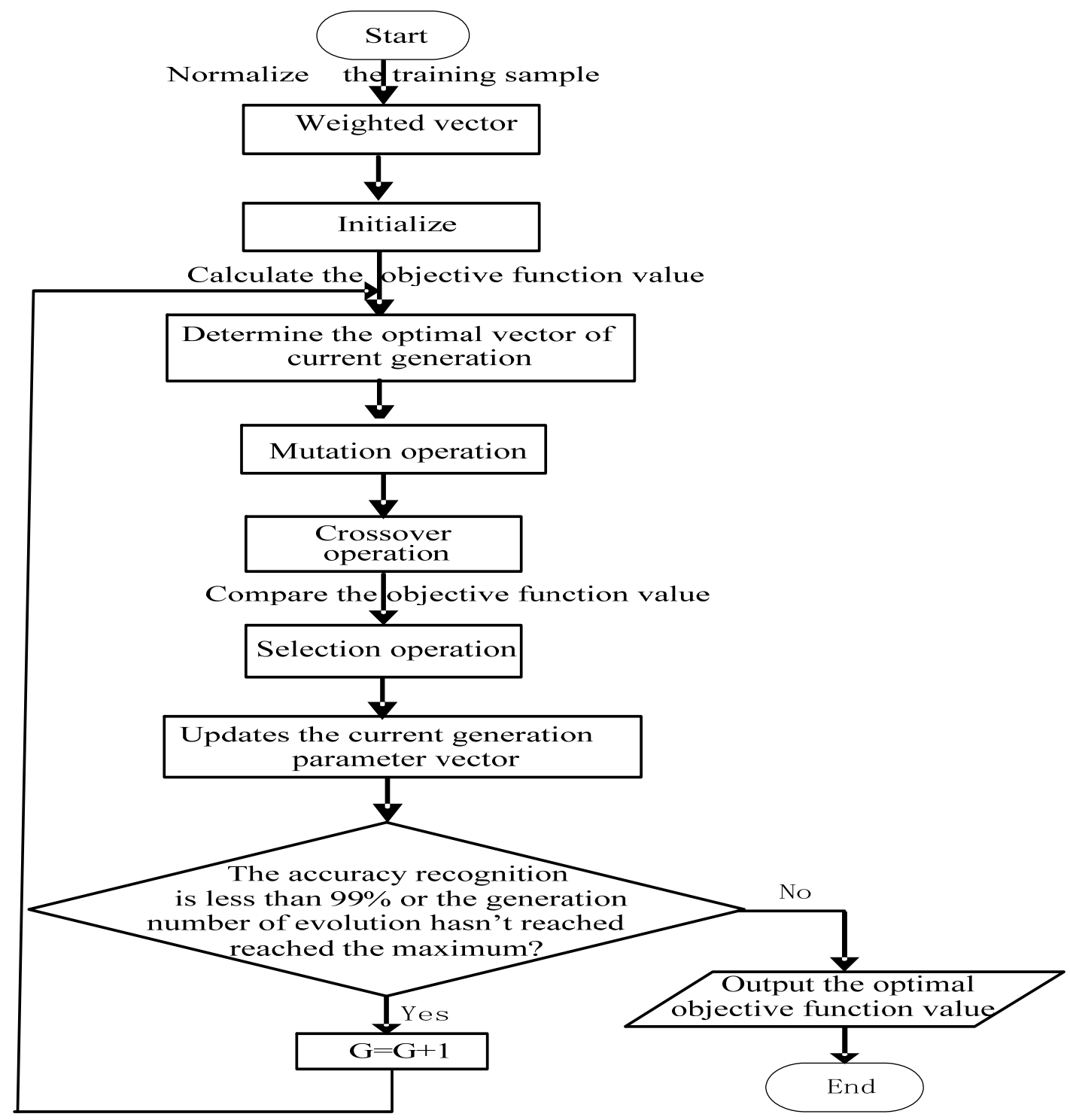

Figure 1. DEPNN Algorithm Flow Chart

Digital image enhancement technology is the basic technology of digital image processing, image enhancement is intended to highlight the human image or part of its interest features for the subsequent image recognition, understanding, the output of other services. PDE-based image processing method of image processing is an important branch, and associated image processing 
content with the benefit of researchers in related fields called common focus of attention. Table 1 The comparison of SNR、PSNR、MSE of the two denoising method.

Table 1. The comparison of SNR、PSNR、MSE of the two denoising method

\begin{tabular}{|c|l|l|l|}
\hline \multirow{2}{*}{ Image } & Model & Local ROF model & $\begin{array}{l}\text { Non local ROF } \\
\text { model }\end{array}$ \\
\hline \multirow{4}{*}{ Composite image } & SNR & 4.892 & 12.347 \\
\cline { 2 - 4 } & PSNR & 24.593 & 28.217 \\
\cline { 2 - 4 } & MSE & 764.517 & 137.357 \\
\hline \multirow{3}{*}{ Barbara image } & SNR & 22.986 & 14.098 \\
\cline { 2 - 4 } & PSNR & 10.165 & 27.486 \\
\cline { 2 - 4 } & MSE & 249.359 & 95.671 \\
\hline
\end{tabular}

\section{Conclusions}

This article compares the system to be introduced and discussed based image denoising partial differential equations, partial differential model focused on the image denoising, image enhancement, etc., for their advantages and disadvantages made corresponding improvement, and thus the simulation. The main idea is the coherence enhancing diffusion model and anisotropic diffusion model combining combines the advantages of the advantages of coherence enhancing diffusion model can handle both at the border and the noise can be well protected and details of anisotropic diffusion model to overcome the blurred edge , complement each other, and achieved certain results in the elimination of image noise, image smoothing. After using the concept of non-local derivative, local ROF model is extended to non-local anisotropic diffusion. Numerical experiments show that non-local model can well remove the noise, and can be well protected the important features of the image edge and texture.

\section{References}

[1] Haralick R M., Shanmugam K and Dinslein L, "Textural features for image classification," IEEE Trans. Systems, Man and Cybernetics, vol.3, pp.610-621,1973.

[2] P.Parveen and B.Thuraisingham, "Face Recognition using Multiple Classifiers," 8th IEEE international Conference on Tools with Artificial Intelligence (ICTAI'06), pp.179-186,2006.

[3] S.Sidhu and D.K.Raahemifar, "Texture Classification using Wavelet Transform and Support Vector Machines," Canndian Conference on Electrical and Computer Engineering, pp.941-944,2005.

[4] Gao Decheng and Wang Jianguo, "Texture Classification Based on Nonsubsampled Directional Filter Banks and Support Vector Machines," 2010 Second Asia-Pacific Conference on Information Processing (APCIP 2010),2010.

[5] Y.Yang and L.K.yang, "Neural Network Based on GA-BP Algorithm and its application in the Protein Secondary Structure Prediction,” Chinese . of Biomedical Engineering (English Edition), vol.15,pp.1-9,2006. 
[6] T.Ji and H.Shi, "Soft Sensor Modeling for Temperature Measurement of Texaco Gasifier Based on an Improved RBF Neural Network, " IEEE international Conference on Information Acquisition,pp.1147-1151,2006.

[7] C.Y.Chang and S.Y.Fu, "Image Classification using a Module RBF Neural Network," 1th International Conference on Innovative Computing,Information and Control (ICICIC'06),pp.270-273,2006.

[8] T.Song, M.Jamshidi, R.R.Lee,et al., “A Novel Weighted Probabilistic Neural Network for MR Image Segmentation," IEEE International Conference on System,Man and Cybernetics, pp.2501-1506,2005.

[9] Y.L.Gao and Z.Z.Zhang, "Modulation Recognition Based on Combined Feature Parameter and Modified Probabilistic Neural Network," 6th World Congress on Intelligent Control and Automation, pp.2954-2958,2006.

[10] D.Q.Yang, S.P.Xiao, and J.F.Jiang, "Texture image recognition based on modified probabilistic neural network," The Fifth International Symposium on Multispectral Image Processing \& Pattern Recognition, vol.6788, pp.1-8, 2007.

[11] L.Rutkowski and S.Member, "Adaptive Probabilistic Neural Networks for Pattern Classification in Time-Varying Environment,'Transactions on Neural Networks, vol.15, pp.811-827,2004.

[12] Y.J.Liu, C.J.Zhao, S.J.Sun,et al., "Texture Recognition Method Research Based on Wavelet Technology, " Proceedings of 2011 IEEE the 18th International Conference on Industrial Engineering and Engineering Management, pp.592-594,2011. 